\title{
Patterns of p73 N-terminal isoform expression and p53 status have prognostic value in gynecological cancers
}

\author{
KERSTIN BECKER ${ }^{1}$, PETR PANCOSKA ${ }^{1}$, NICOLE CONCIN ${ }^{3}$, KELLY VANDEN HEUVEL ${ }^{1}$, \\ NEDA SLADE ${ }^{1,4}$, MARGARET FISCHER $^{2}$, EVA CHALAS $^{2}$ and UTE M. MOLL ${ }^{1}$ \\ ${ }^{1}$ Department of Pathology, ${ }^{2}$ Division of Gynecologic Oncology, Departments of Obstetrics, Gynecology \\ and Reproductive Medicine, State University of New York at Stony Brook, Stony Brook, NY, USA
}

Received April 4, 2006; Accepted June 13, 2006

\begin{abstract}
The goal of this study was to determine whether patterns of expression profiles of p73 isoforms and of p53 mutational status are useful combinatorial biomarkers for predicting outcome in a gynecological cancer cohort. This is the first such study using matched tumor/normal tissue pairs from each patient. The median follow-up was over two years. The expression of all $5 \mathrm{~N}$-terminal isoforms (TAp73, $\Delta \mathrm{Np} 73$, $\Delta N^{\prime} p 73$, Ex2p73 and Ex2/3p73) was measured by real-time RT-PCR and p53 status was analyzed by immunohistochemistry. TAp73, $\Delta \mathrm{Np} 73$ and $\Delta \mathrm{N}^{\prime} \mathrm{p} 73$ were significantly upregulated in tumors. Surprisingly, their range of overexpression was age-dependent, with the highest differences $\delta$ (tumornormal) in the youngest age group. Correction of this age effect was important in further survival correlations. We used all 6 variables (five p73 isoform levels plus p53 status) as input into a principal component analysis with Varimax rotation (VrPCA) to filter out noise from non-disease related individual variability of p73 levels. Rationally selected and individually weighted principal components from each patient were then used to train a support vector machine (SVM) algorithm to predict clinical outcome. This SVM algorithm was able to predict correct outcome in 30 of the 35 patients. We use here a mathematical tool for pattern recognition that has been commonly used in e.g. microarray data mining and apply it for the first time in a prognostic model. We find that PCA/ SVM is able to test a clinical hypothesis with robust statistics and show that p73 expression profiles and p53 status are
\end{abstract}

Correspondence to: Dr Ute M. Moll, Department of Pathology, Stony Brook University, Stony Brook, NY 11794-869, USA

E-mail: umoll@ notes.cc.sunysb.edu

Present addresses: ${ }^{3}$ Department of Obstetrics and Gynecology, Innsbruck Medical University, Innsbruck, Austria; ${ }^{4}$ Department of Molecular Medicine, Ruder Boskovic Insitute, Zagreb 10 000, Croatia

Abbreviations: PCA, principal component analysis; SVM, support vector machine

Key words: ovarian cancer, p53, p73, isoforms, survival useful prognostic biomarkers that differentiate patients with good vs. poor prognosis with gynecological cancers.

\section{Introduction}

The p53 gene family consists of p53, p63 and p73. p73 shares substantial homology with $\mathrm{p} 53$. In response to DNA damage or deregulated oncogenes, full-length p 73 can activate many p53 target genes, thereby inducing cell cycle arrest or apoptosis (1-7). In mice, recent genetic evidence found some support for a helper tumor suppressor role of p73 once p53 is impaired. While p73-/- mice completely lack a cancer phenotype (8), double heterozygous p53+/-, p73+/- mice have a higher tumor burden compared to p53+/- mice (9). In human cancers, however, p73 is not a classical tumor suppressor. The virtual absence of p73 inactivating mutations (10), combined with tumor-associated overexpression of wild-type p73 isoforms including TAp73 in human cancers are in sharp contrast to the notion of a tumor suppressor function. One explanation lies in the fact that, unlike p53, the p73 gene gives rise to multiple isoforms with different and often opposing functions (Fig. 2A). Whereas the full-length isoform TAp73 largely mimics p53 function, four additional $\mathrm{NH}_{2}$-terminally truncated isoforms, $\Delta \mathrm{Np} 73, \Delta \mathrm{N}$ 'p $73, \mathrm{Ex} 2 \mathrm{p} 73$ and Ex23p73, act as dominant negative inhibitors of both TAp73 and wild-type p53 (11-13). The isoforms TAp73, $\Delta N^{\prime}$ p73, Ex2p73 and Ex2/ $3 \mathrm{p} 73$ are all generated from $\mathrm{P} 1$ promoter-derived transcripts via alternative exon splicing (14-16). In contrast, $\Delta \mathrm{Np} 73$ is generated by the P2 promoter in intron 3 (17). Of note, the $\Delta \mathrm{N}^{\prime} \mathrm{p} 73$ and Np73 transcripts encode the same protein due to a premature translational stop in the $5^{\prime}$ region of exon $3^{\prime}$ in $\Delta \mathrm{N}^{\prime} \mathrm{p} 73$. Importantly, all $\mathrm{NH}_{2}$-terminally truncated p73 isoforms lack all or most of the transactivation domain and are therefore collectively called $\Delta \mathrm{TAp} 73 \mathrm{~s}$. They retain their DNAbinding and tetramerization competence, which enables them to inhibit TAp73 and wild-type p53 function by direct promoter competition as well as by heterocomplex formation $(13,18-20)$.

Experimental and clinical evidence is mounting that $\Delta \mathrm{TAp} 73 \mathrm{~s}$ might indeed act as biologically relevant oncogenes in human cancers $(17,21,22)$. Moreover, recent clinical studies support this idea by identifying $\Delta \mathrm{Np} 73$ as a significant independent factor predicting poor prognosis in neuroblastoma and lung cancer patients $(23,24)$. Of note, most of the previous p73 expression studies in primary human cancers did not 
discriminate between TAp73 and $\Delta \mathrm{TAp} 73$ isoforms, nor did they quantitate expression levels of different p 73 isoforms (25-28). Therefore, we recently undertook a large prognostic study to determine expression levels of all $\Delta \mathrm{TAp} 73$ and TAp73 products in 100 primary ovarian carcinomas using isoformspecific quantitative real-time RT-PCR (29). Tumor expression levels were comapred to a pool of 48 unmatched normal tissues. Of note, $95 \%$ of ovarian cancers strongly overexpressed $\Delta \mathrm{N}^{\prime} \mathrm{p} 73$, and a small subgroup of tumors overexpressed $\Delta \mathrm{Np} 73$, while about one-third of tumors also exhibiting concomitant up-regulation of TAp73. We observed a trend for better overall survival in patients with low expression of $\Delta \mathrm{N}^{\prime} \mathrm{p} 73 / \mathrm{Np} 73$, compared to patients with high expression. Our recent followup study that was expanded to 122 ovarian cancer patients was able to demonstrate significant correlations between high $\Delta \mathrm{N}^{\prime} \mathrm{p} 73 / \mathrm{Np} 73$ expression and poor recurrence-free and overall survival, albeit only within the mutant p53 subgroup of ovarian cancers (30).

Here we present the first prognostic study of gynecological cancers with matched tumor/normal tissue pairs, based on quantitative expression of all five $\mathrm{N}$-terminal p73 isoforms, p53 status and a median follow-up of more than two years. We used all 6 molecular variables (p73 isoform levels plus p53 status) as input into the PCA/Varimax algorithm to test whether they could serve as combinatorial biomarkers for predicting outcome in this cohort. Using the resulting principal components, the SVM was able to correctly predict outcome in 30 of the 35 patients.

\section{Materials and methods}

Tissues. Forty-seven women presenting to the gynecologic oncology service at Stony Brook University Hospital between November 2000 and February 2003, with known or suspected gynecologic cancers and for whom surgery was the planned therapeutic intervention, were invited to participate in prospective tissue collection. They consented to sampling of normal and malignant tissue during surgery in strict accordance with the Institutional Review Board approved protocol. From each patient, tumor tissue was obtained from ovary or endometrium or tumor-replaced omentum, and normal tissue was obtained from peritoneum or in some cases from macroscopically normal ovary. After resection, tissues were immediately snap frozen in liquid nitrogen and stored at $-80^{\circ} \mathrm{C}$ until used. For conventional clinicopathological correlations, all 47 patients were used (Table I). For the PCA/Varimax analysis (VrPCA), a subset of 35 patients for whom complete clinical follow-up data and adequate sampling for p73 isoform measurements were available, were used. A scheme of the 5 isoforms (TAp73, $\Delta \mathrm{Np} 73, \Delta \mathrm{N}^{\prime} \mathrm{p} 73, \mathrm{Ex} 2 \mathrm{p} 73$ and Ex2/3p73) is shown in Fig. 2A. For p73 expression analysis, RNA extraction, cDNA synthesis, real-time RT-PCR experiments and raw data analyses were performed as previously described (29). p53 status was determined by immunostaining with DO-1 monoclonal antibody (Calbiochem) on paraffin tissue sections from the same tumor mass that was used for RNA extraction, as described (31). Tumors were scored as mutant if they showed $>10 \%$ strongly positive tumor cell nuclei. In ovarian, endometrial and primary peritoneal carcinoma, a statistically significant association between p53 immunostaining and p53
Table I. Clinicopathological correlations of 43 patients from this study.

\begin{tabular}{|c|c|c|c|}
\hline & & $\mathrm{n}$ & $\%$ \\
\hline \multirow[t]{5}{*}{ Diagnosis } & Primary ovarian cancer & 32 & 68.0 \\
\hline & Primary peritoneal cancer & 8 & 17.0 \\
\hline & Ovarian borderline tumor & 4 & 8.0 \\
\hline & Endometrial cancer & 3 & 6.0 \\
\hline & Total & 47 & 100.0 \\
\hline \multirow[t]{5}{*}{ FIGO stage } & I & 6 & 14.0 \\
\hline & II & 4 & 9.0 \\
\hline & III & 23 & 53.0 \\
\hline & IV & 10 & 23.0 \\
\hline & Total & 43 & 100.0 \\
\hline \multirow[t]{4}{*}{ Grading } & 1 & 3 & 7.0 \\
\hline & 2 & 3 & 7.0 \\
\hline & 3 & 37 & 86.0 \\
\hline & Total & 43 & 100.0 \\
\hline \multirow[t]{7}{*}{ Cell type } & Serous & 27 & 62.8 \\
\hline & Endometrioid & 4 & 9.3 \\
\hline & Clear cell & 4 & 9.3 \\
\hline & Sarcoma & 1 & 2.3 \\
\hline & Anaplastic & 1 & 2.3 \\
\hline & Mixed & 6 & 14.0 \\
\hline & Total & 43 & 100.0 \\
\hline
\end{tabular}

The 4 ovarian borderline tumors were excluded from stage, grade and cell type classification.

mutations [i.e. $\mathrm{p}=0.0002$ (32)], and a correlation between p53 mutations as well as p53 overexpression to shorter patient survival is well supported by many studies [e.g. (32-36)].

Clinical data. Ovarian cancer patients underwent total hysterectomy (TH) with bilateral salpingo-oophorectomy (BSO) and tumor debulking, followed by 6 cycles of Taxol/ Carboplatin. Borderline tumors were treated with TH/BSO only. The 3 endometrial cancer patients underwent TH/BSO. The median age at diagnosis was 61 years (range 38-87). Table I lists the clinicopathological characteristics of our patient cohort. For follow-up, a combination of physical exam, CA125 serum levels and imaging was used to determine status. Continuous increase in CA125 levels was considered a chemical recurrence. For survival analysis, all cancers (except 4 borderline tumors) of stages I and II were combined into the early stage group $(10 / 43 ; 23 \%)$ and stages III and IV were combined into the advanced stage group $(33 / 43 ; 77 \%)$. To classify tumor grade, the widely used system based on architectural and nuclear grade and mitotic activity was applied. Grades 1 and 2 were pooled into one group and compared to grade 3 tumors. 
The median time of follow-up was 839 days (range 342724 days). Patients were classified into 3 follow-up groups, i.e. free of disease (group I, 43\%; 20/46 patients), alive with disease (group II, 22\%; 10/46 patients) and dead of disease (group III, 35\%; 16/46 patients). For our prognostic model, group I was the good prognostic group. Groups II and III were pooled and represented the bad prognostic group. The median time of follow-up was 650 days for group I, and 757 days for pooled groups II and III (range 34-2724 days). For validation of our patient cohort, survival probabilities were also calculated by the product limit method of Kaplan-Meier. Differences between groups were tested using the log-rank test. The results were analyzed for end points of recurrencefree and overall survival. Overall survival was defined as the time between date of surgery and death. Live patients were censored at the last date they were known to be alive. In summary, data for 35 patients were useable for final analysis. (Of the initially 47 patients with clinicopathological data, 43 patients were classified according to FIGO stages, while 4 patients had borderline tumors. One of the 47 patients was lost to follow-up. Of the remaining 46 patients, the complete set of p73 isoforms and p53 mutational status could be determined in a total of 35 patients). Recurrence-free survival was calculated from the date of surgery until the date when progressive disease, relapse or death was reported, whichever occurred first. Patients who had not experienced any unfavourable event were censored at the last date they were known to be alive.

Statistical analyses. Descriptive statistics were used to obtain frequency tables for all categorical variables. Means and standard deviations were calculated for continuous variables. Since some normal tissues lacked detectable p73 isoform expression (especially $\Delta \mathrm{N}^{\prime} \mathrm{p} 73$ ), which precluded the calculation of a meaningful tumor/normal ratio, we expressed the amount of p73 isoform up-regulation as the difference between the two values ( $\delta=$ tumor-normal absolute copy numbers for each isoform). These values were age-corrected for $\delta \mathrm{TAp} 73, \delta \Delta \mathrm{Np} 73$ and $\delta \Delta \mathrm{N}^{\prime} \mathrm{p} 73$, and now called $\delta^{*}$ (see Results).

As the data distribution was non-Gaussian, non-parametric tests were applied for further analyses. The Mann-Whitney U-test was used when comparing continuous variables between two different groups, while the Kruskal-Wallis test was used when analysing variables with more than two categories. To analyze the difference of p73 isoform levels between tumor and normal tissue, the Wilcoxon test was applied. P-values $<0.05$ were considered statistically significant.

To correlate clinical outcome with p73 isoform levels, we constructed a data matrix where each of the 35 patients (row) was characterized by six molecular variables (columns): $\delta^{*} \mathrm{TA}$, $\delta^{*} \Delta \mathrm{N}, \delta^{*} \Delta \mathrm{N}^{\prime} \mathrm{p} 73$, normalized $\delta \mathrm{Ex} 2, \delta \mathrm{Ex} 2 / 3 \mathrm{p} 73$ and binary encoded p53 status ( $0 \mathrm{wt}, 1$ mutated). This $35 \times 6$ matrix was then used as input into a principal component analysis (PCA) (37), followed by Varimax rotation (38) of the principal component solution. We applied PCA and Varimax algorithm (VrPCA), implemented in the SPSS software (SPSS Inc., v. 11.0), which resulted in five principal components (c1-5) for each of the 35 patients. These new, transformed characteristics were then used in the development of a model

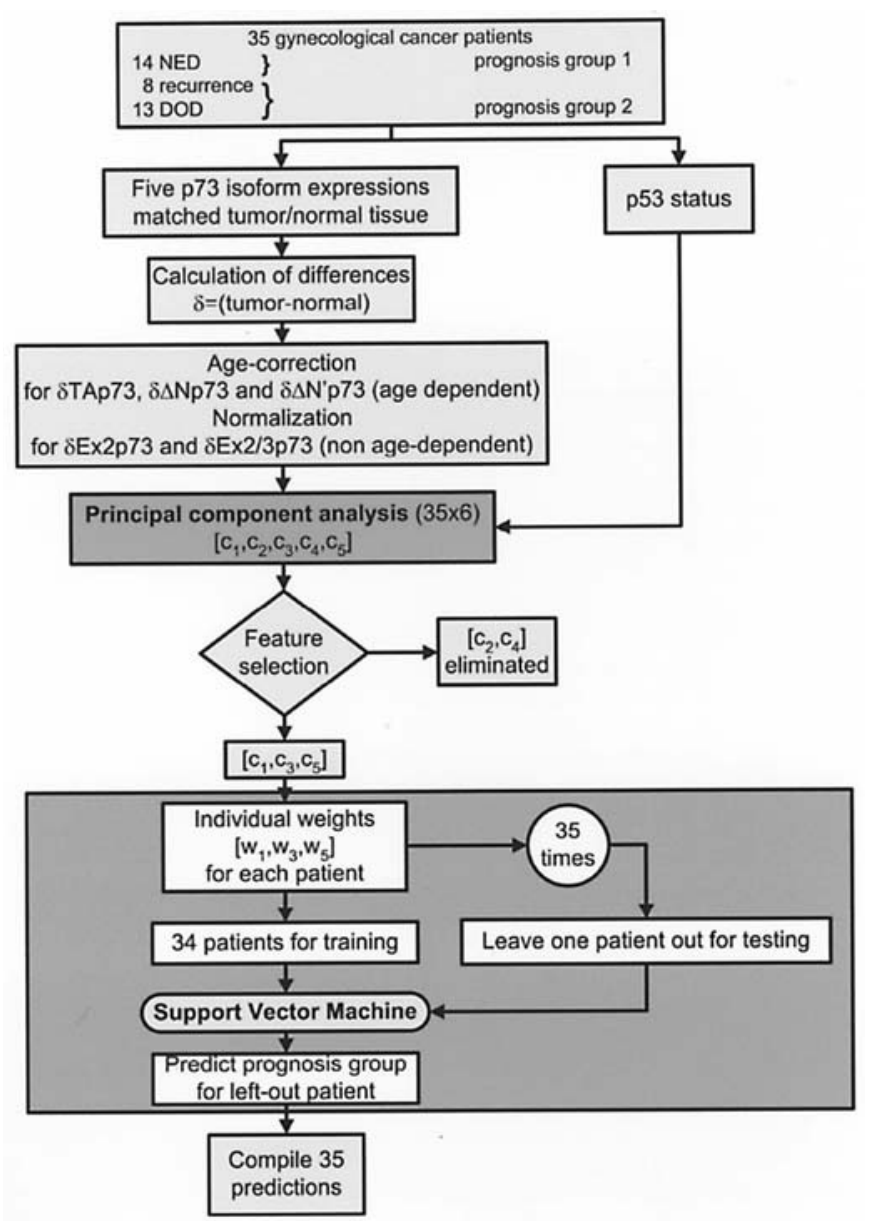

Figure 1. Flow-diagram of steps used for the PCA-based prognostic model.

predicting prognostic categories. Our prognostic model was based upon a support vector machine (SVM) algorithm, implemented in the Weka v. 3.4 software (39). To obtain the best predictive model, we systematically performed a complete 35-fold leave-one-out testing on each of the possible combinations of PCA weights (see below) among the five principal components. For example, first, PCA weights of only one of the five components for each patient were tested, then all possible paired combinations of components, then all possible triplet combinations etc., until the best predictive combination was found. This turned out to be the combination of components 1, 3 and 5 (see Results). Fig. 1 summarizes these steps in a flow diagram.

\section{Results}

Clinicopathological correlations. To validate the reliability of the survival data of our cohort, we first analyzed the prognostic impact of the well established clinical parameters 'stage' and 'grade' in this patient group. As expected, patients presenting with stages I or II had a significantly longer relapse-free survival (median 1331 vs. 677 days; $\mathrm{p}=0.0024$ ) and a trend towards longer overall survival (median 1340 vs. 1009 days; $\mathrm{p}=0.083$ ) than patients with stages III or IV (Fig. 8 and data not shown). Conversely, patients with well (grade 1) or moderately (grade 2) differentiated tumors showed a significantly longer relapse-free survival $(\mathrm{p}=0.0166)$ and a trend towards longer 
Table II. Descriptive statistics of p73 isoform expression levels and comparison between tumor/matched normal pairs by the Wilcoxon test.

\begin{tabular}{|c|c|c|c|c|c|c|c|c|}
\hline p73 isoform & & $\mathrm{n}$ & Mean & Median & $\begin{array}{l}\text { Standard } \\
\text { deviation }\end{array}$ & Min & Max & $\begin{array}{c}\text { Significance in } \\
\text { Wilcoxon test }(\mathrm{p})\end{array}$ \\
\hline \multirow[t]{2}{*}{ TAp73 } & tu & 36 & 12574.59 & 862.35 & 24522.28 & 0 & 106563 & 0.000 \\
\hline & norm & 36 & 307.33 & 73.05 & 410.92 & 0 & 1552 & \\
\hline \multirow[t]{2}{*}{$\Delta \mathrm{Np} 73$} & tu & 36 & 267.19 & 21.95 & 804.31 & 0 & 4603 & 0.025 \\
\hline & norm & 36 & 57.58 & 19.5 & 133.32 & 0 & 742 & \\
\hline \multirow[t]{2}{*}{$\Delta N^{\prime}$ p73 } & tu & 36 & 496.73 & 38.25 & 1273.17 & 0 & 7043.5 & 0.000 \\
\hline & norm & 36 & 6.54 & 0 & 28.10 & 0 & 162.8 & \\
\hline \multirow[t]{2}{*}{ Ex2p73 } & tu & 36 & 118.33 & 30.65 & 191.28 & 0 & 851 & 0.859 \\
\hline & norm & 36 & 103.22 & 40.35 & 169.53 & 0 & 695 & \\
\hline \multirow[t]{2}{*}{ Ex2/3p73 } & tu & 36 & 39863.07 & 5873.5 & 86500.07 & 642 & 392911 & 0.987 \\
\hline & norm & 36 & 39440.50 & 4205.5 & 79649.88 & 543.1 & 344335 & \\
\hline
\end{tabular}

overall survival $(\mathrm{p}=0.1058)$ than patients with poorly differentiated (grade 3) tumors (Fig. 8 and data not shown). We also found significant correlations between survival and $\mathrm{EC}_{50}$ values of CA125 and between a poor drop of CA125 and a high $\Delta$ TAp73/TAp73 ratio (Figs. 9-11). No significant correlation was found between p73 isoform levels and FIGO stage, grade, histological type or p53 status.

Fifty percent (17/34) of the tumors harboured p53 mutations, as determined by immunoperoxidase staining. p53 mutations were more common in primary peritoneal cancers $(7 / 8 ; 88 \%)$ than in primary ovarian cancers $(9 / 20 ; 45 \%)$ and endometrial cancers $(1 / 3 ; 33 \%)$, while borderline tumors were wild-type for $\mathrm{p} 53$. No significant association was detectable between p53 mutational status and tumor stage or grade. However, patients with wild-type p53 displayed a borderline significance towards longer relapse-free survival than patients with p53 mutations (median 1109 vs. 670 days; $\mathrm{p}=0.0573$ ).

TAp73, $\triangle N p 73$ and $\triangle N^{\prime} p 73$ are up-regulated in tumors. In 36 patients, expression levels of all five p73 isoforms could be determined by real-time RT-PCR in tumor and matched normal tissues. After normalization of each sample to its own 28S rRNA transcript level, data were expressed as absolute copy numbers per unit input of total RNA (154 ng). For TAp73, $\Delta \mathrm{Np} 73$ and $\Delta \mathrm{N}^{\prime} \mathrm{p} 73$, expression was significantly higher in tumor samples compared to their matched normal tissues (Table II and Fig. 2B). In contrast, Ex2p73 and Ex2/ 3 p73 were frequently not up-regulated, while a subgroup of tumors exhibited up- or down-regulation, clearly distinguishing their behavior as separate from TAp $73, \Delta \mathrm{Np} 73$ and $\Delta \mathrm{N}^{\prime} \mathrm{p} 73$ (Table II and Fig. 3B). This will be subsequently reflected by their major contribution to component 1 (see below).

Tumor-associated TAp73, $\triangle N p 73$ and $\Delta N^{\prime} p 73$ up-regulation is age-dependent. The calculated raw differences were plotted as a function of patient age for each isoform (Fig. 3). The analysis of these plots revealed that the maximum ranges of TAp $73, \Delta N p 73$ and $\Delta N^{\prime}$ p 73 , but not of Ex2p73 and Ex2/ $3 p 73$, systematically decreased with increasing patient age, thus were age-dependent (Fig. 3A left panels, and 3B). This resulted in large range variances in the younger age groups in contrast to a compressed-appearing variance for the older age groups, which distorted comparisons between age groups. Importantly, however, within each age group, the relative variance in relation to its own mean was comparable, which justified the following age adjustments for values. In order to render relative variances of different age groups comparable with each other, we divided patients into age groups of 10 years starting from age 35 , and selected the maximum $\delta \mathrm{X}$ expression for each age group $\left(X=\mathrm{TAp} 73, \Delta \mathrm{Np} 73, \Delta \mathrm{N}^{\prime} \mathrm{p} 73\right)$. These maximum values were then least-square fitted by the sigmoidal function:

$$
\phi(\text { age })=\frac{\delta_{\max }}{\left(1+\left(\frac{\text { age }}{t_{1 / 2}}\right)^{k}\right)}
$$

$t_{1 / 2}$ is the age at which the range of the observed $\delta$ values is one half of the maximum range observed within the patient cohort. $k$ is the slope, i.e. the rate at which $\delta$ range decreases with age. The choice of sigmoidal functions for age correction was empirical but is the most natural choice for a correction function (Fig. 3A). It avoids problems with unrealistic infinity values for limits, as would be seen e.g. with linear or quadratic functions. Thus, for every age group, there is a maximum range of expression levels that decreases sigmoidally with the age. We approximate this maximum range of isoform expression by the maximum experimentally observed expression level for every age group. As validation serves the fact that these maximum levels can be fitted by a smooth function. If this approximation of the range were vastly incorrect, we could not define these smooth functional 
A
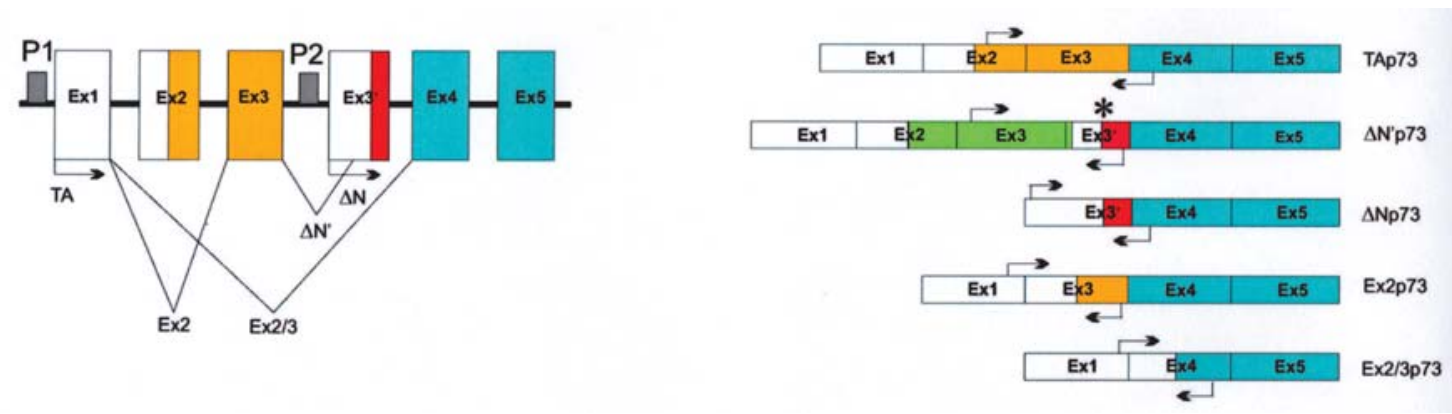

$\mathrm{B}$
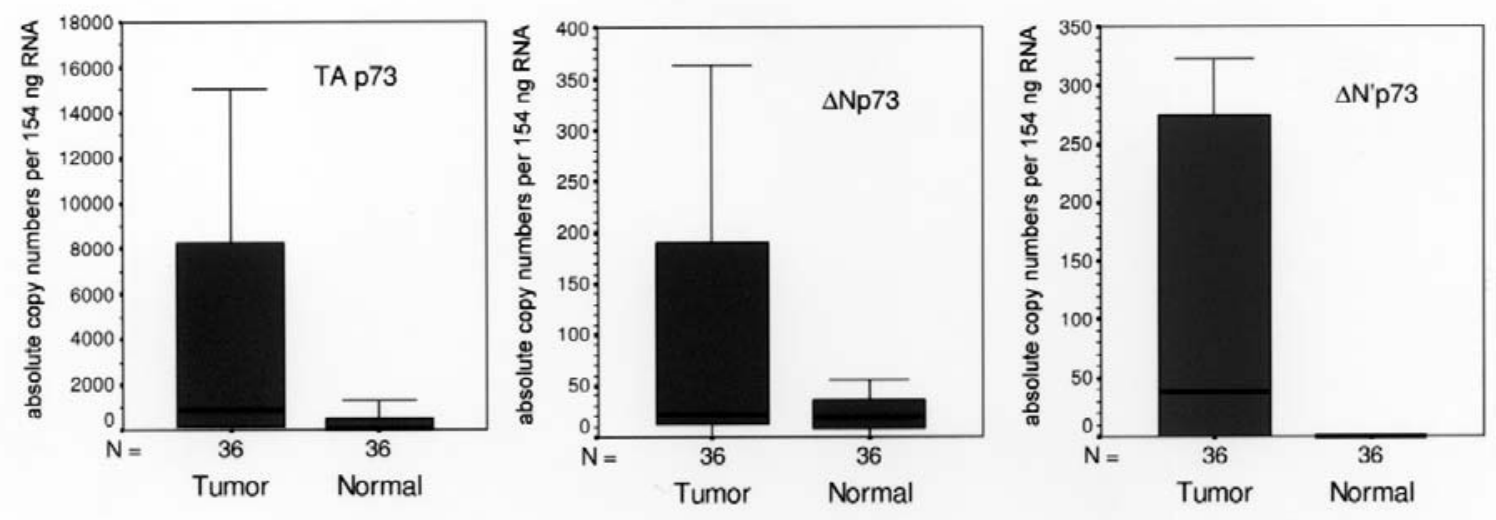

Figure 2. A, The complete set of $\mathrm{NH}_{2}$-terminal transcripts encoding TAp73 and $\triangle \mathrm{TAp} 73$ isoforms. Left, gene architecture of the $\mathrm{NH}_{2}$ terminus of $T P 73$. The various isoforms are indicated. Arrows indicate transcriptional start sites. Right, positions of reverse transcription (RT)-PCR primers for the various p73 isoforms used in this study. White, untranslated sequences; yellow, transactivation domain; red, exon 3' derived coding sequences; blue, DNA-binding domain; green, translated (but non-productive sequence) of $\Delta \mathrm{N}^{\prime} \mathrm{p} 73$ that undergoes a premature stop in the $5^{\prime}$ untranslated region of exon $3^{\prime}$. The star indicates the used second translational start site in $\Delta \mathrm{N}^{\prime} \mathrm{p} 73$. In $\Delta \mathrm{Np} 73$, Ex2p73, and Ex2/3p 73 translational start sites are indicated by color borders. B, TAp $73, \Delta N p 73$ and $\Delta N^{\prime}$ p73 are up-regulated in tumors compared to their matched normal tissue. Boxplots comparing expression levels of TAp73, $\Delta N p 73$ and $\Delta N^{\prime}$ p73 between tumors and matched normal tissues. The line within the boxes indicates the median. The upper edge of the boxes represents the 75 th percentile, the lower edge the 25 th percentile. Whiskers demarcate the 10th and 90th percentile. The range is shown as a vertical line. Outliers (defined as 1.5 -fold above or below the 75th and 25th percentile values) are not shown.

boundaries from our experimental data. Thus, the result of this age correction is that these maximum expression ranges are brought to equal footing for all age groups in the study. This then allows to compare the individual expression levels and is equivalent to using relative expression levels for each age group (calculated as percentage of the maximal expression level observed experimentally for each age group). All p73 isoforms follow the same sigmoidal function (Fig. 3A and data not shown). No correlation existed between age and the length of overall survival. The raw $\delta X$ values were then converted into age-corrected $\delta^{*}$ values $\left(\delta^{*} X=\delta X / \phi(\right.$ age $\left.)\right)$. This normalized the values by generating an even distribution across ages (Fig. 3A right panels). Table III shows the descriptive statistics for raw and age-corrected $\delta$ values for $\Delta \mathrm{Np} 73, \Delta \mathrm{N}^{\prime} \mathrm{p} 73$ and TAp73 in the entire cohort.

In contrast, Ex2p73 and Ex2/3p73 showed no systematic tumor-associated up-regulation and no age-dependence (Fig. 3B). Moreover, although Ex2p73 and Ex2/3p73 were frequently not up-regulated, a subgroup of tumors exhibited up- or down-regulation, clearly distinguishing their behavior as separate from TAp73, $\Delta \mathrm{Np} 73$ and $\Delta \mathrm{N}^{\prime} \mathrm{p} 73$ (Table II and Fig. 3B). To compensate for big variances in their raw $\delta$ values (Table II) and to bring them into the range of values comparable to the age-corrected $\delta \mathrm{TA}, \delta \Delta \mathrm{N}$ and $\delta \Delta \mathrm{N}^{\prime} \mathrm{p} 73$, we normalized each patient's $\delta$ Ex 2 and $\delta E x 2 / 3 p 73$ values by dividing with the respective maximum $\delta$ from the entire group of 35 patients (Fig. 3B).

PCA-based prognostic modelling. We hypothesized that our failure to observe a direct correlation between $\mathrm{p} 73$ expression levels and prognosis was due to an additional hidden component of each variable that was individual for each patient. In order to filter out this background of individual variances and to extract the crucial information that is disease related, we developed the following model based on principle component analysis with Varimax rotation (VrPCA). Fig. 4 
A

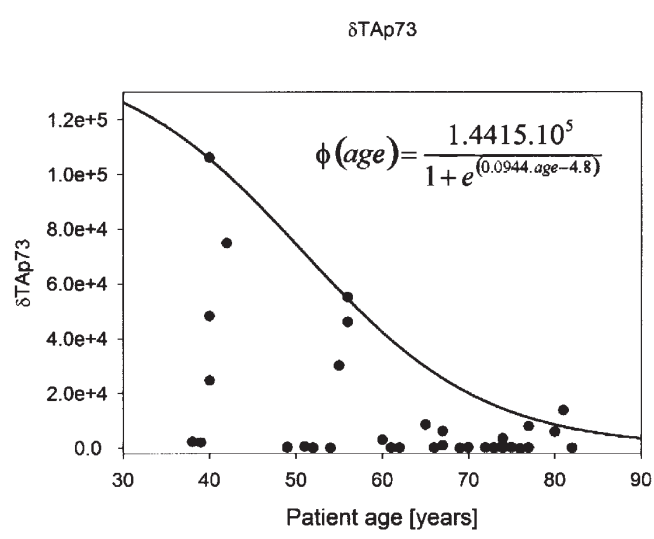

$8 \Delta \mathrm{Np} 73$

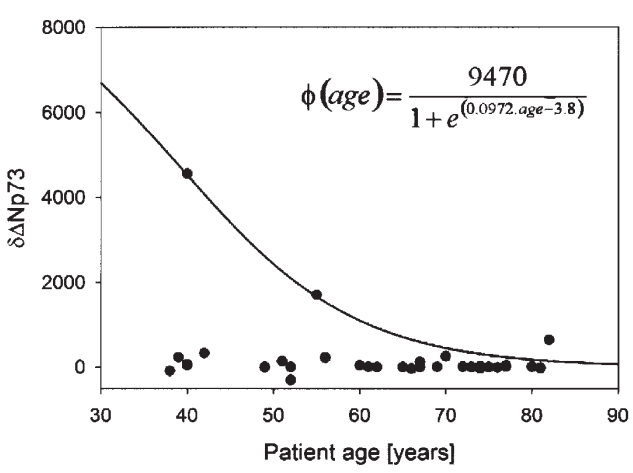

$\delta \Delta N^{\prime} p 73$

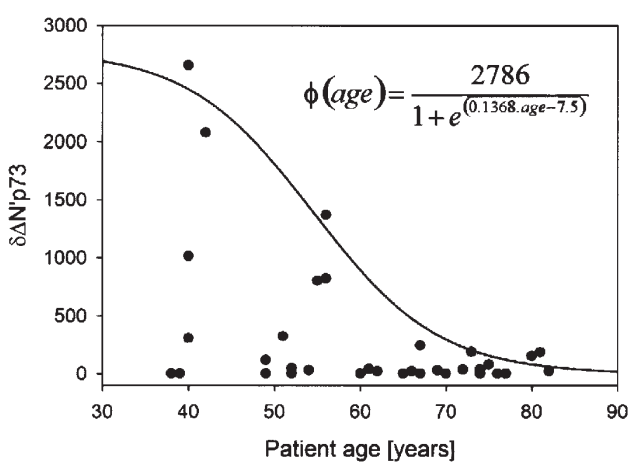

B

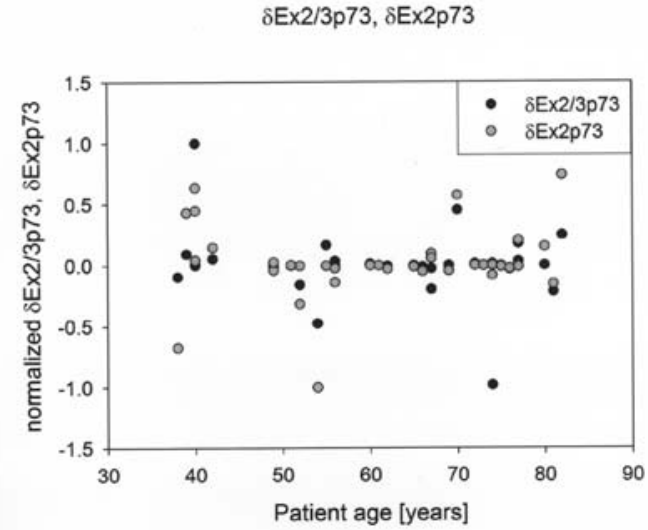

depicts its concept. The total information theoretically available about a given patient (patient $1,2 \ldots . \mathrm{N}$ ) is presented by a large sphere, which contains within it the patient's diseasespecific part, shown as a blue 3-dimensional ellipsoid re-

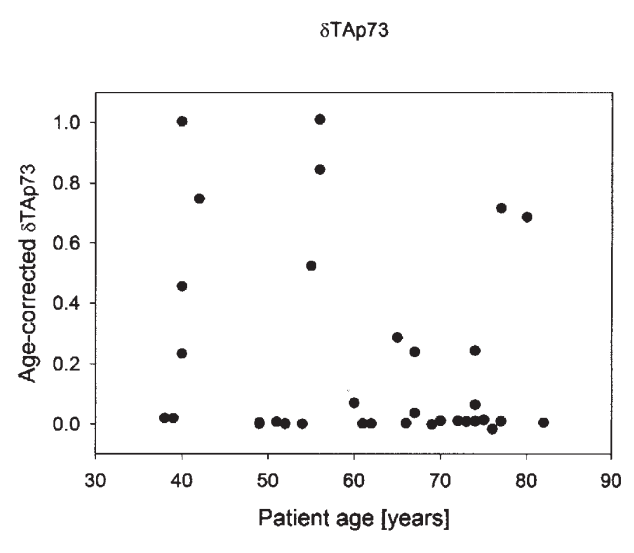

$\delta \Delta N p 73$

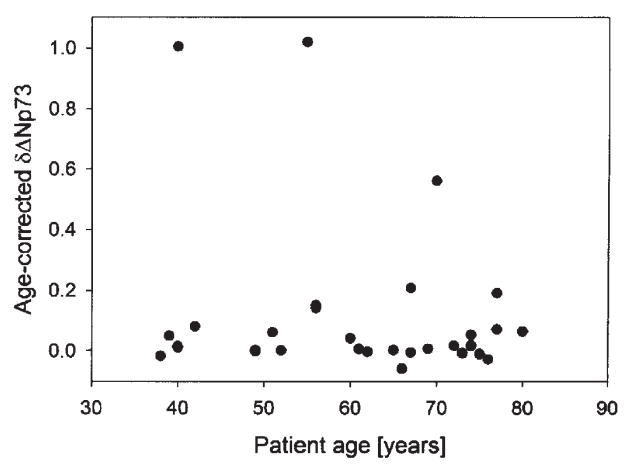

$\delta \Delta \mathrm{N}$ 'p73

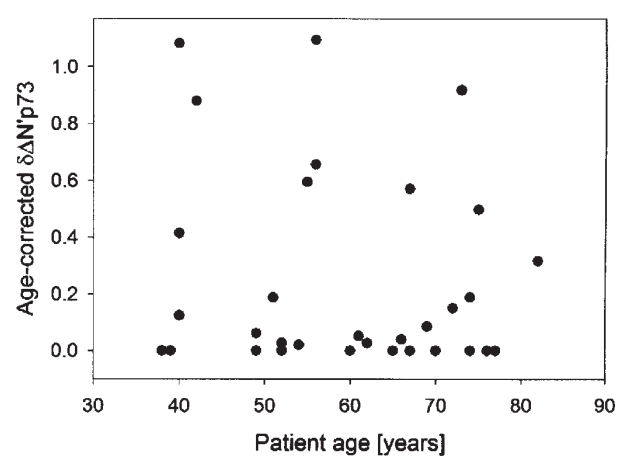

Figure 3. A, The $\delta$ values of TAp73, $\Delta N p 73$ and $\Delta N^{\prime}$ p73 levels of all 35 patients are plotted as a function of age and then least square fitted by sigmoidal curves (left panels). After age-correction according to the respective formula shown above each sigmoidal curve, $\delta^{*}$ values of TAp73, $\Delta \mathrm{Np} 73$ and $\Delta \mathrm{N}$ 'p73 display an even distribution across all ages (right panels). B, Ex2p73 and Ex2/3p73 show no age-dependence. Moreover, although Ex2p73 and Ex2/3p73 were frequently not up-regulated, a subgroup of tumors exhibited up- or down-regulation, distinguishing their behavior as separate from TAp73, $\Delta \mathrm{Np} 73$ and $\Delta \mathrm{N}^{\prime} \mathrm{p} 73$.

sembling a rugby ball. VrPCA helps us to identify the common, tumor-related information shared by all 35 patients, generating a consensus 'rugby ball'. The shape of the individual patient 'rugby ball' is now defined by 3 independent axes of object 
Table III. Descriptive statistics of raw and age-corrected $\delta$ values for TAp $73, \Delta N p 73$ and $\Delta N^{\prime}$ p73 expression levels in the entire cohort.

\begin{tabular}{lrcrrrr}
\hline & \multicolumn{1}{c}{ Raw } & $\begin{array}{c}\text { Age corrected } \\
\delta \text { TAp73 }\end{array}$ & $\begin{array}{c}\text { Raw } \\
\delta \Delta \text { NAp73 }\end{array}$ & $\begin{array}{c}\text { Age corrected } \\
\delta \Delta \text { Np73 }\end{array}$ & $\begin{array}{r}\text { Raw } \\
\delta \Delta \mathrm{N}^{\prime} p 73\end{array}$ & $\begin{array}{c}\text { Age corrected } \\
\delta \Delta \mathrm{N} \text { 'p73 }\end{array}$ \\
\hline $\mathrm{N}$ & 36 & 36 & 36 & 36 & 36 & 36 \\
Mean & 12267.25 & 0.25 & 209.62 & 0.20 & 490.18 & 1.94 \\
Median & 735.45 & 0.02 & 5.65 & 0.01 & 34.70 & 0.07 \\
SD & 24389.12 & 0.41 & 814.15 & 0.77 & 1275.06 & 9.50 \\
Minimum & -206.20 & -0.02 & -593.00 & -0.33 & 0.00 & 0.00 \\
Maximum & 106032.00 & 1.76 & 4547.00 & 4.42 & 7043.50 & 57.27 \\
\hline
\end{tabular}

\section{Patient 1}

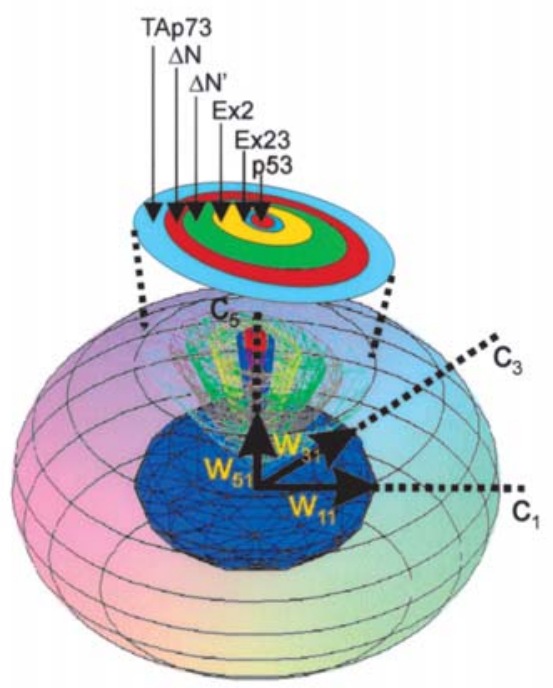

Patient 2

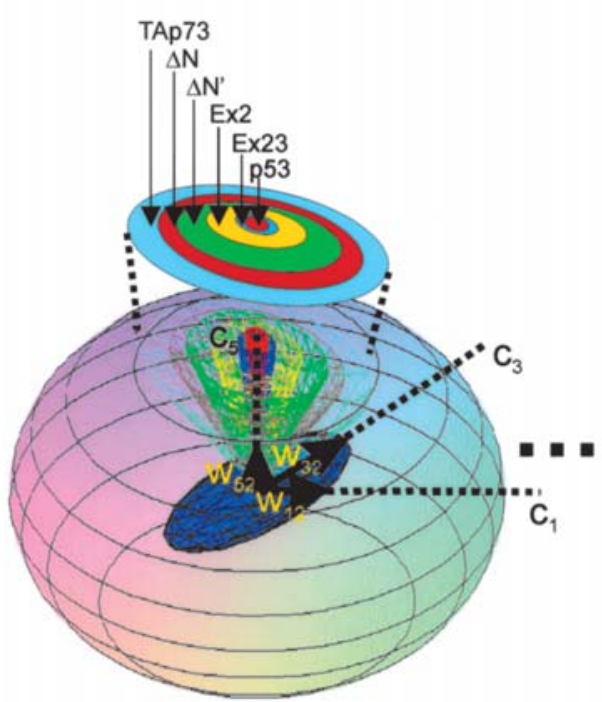

Patient $N$

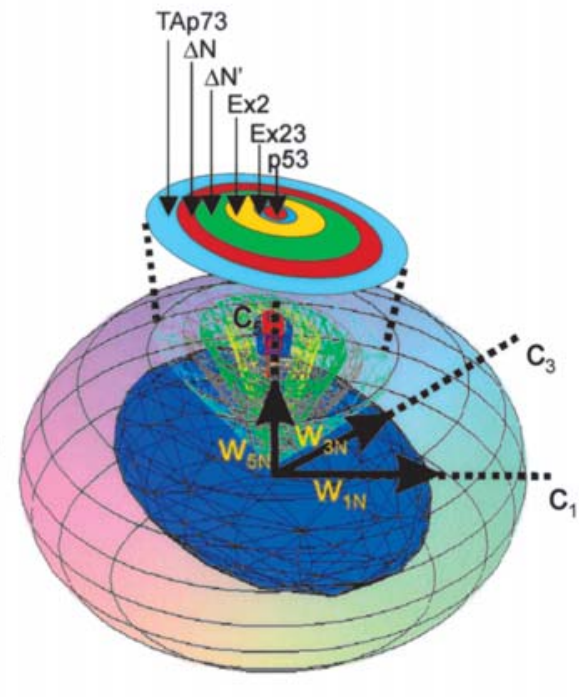

Figure 4. Concept of disease-related (blue 'rugby ball') and individual (outside of 'rugby ball' but within the sphere) contributions to the total information (sphere) on a given patient. Hypothetical patients 1,2 and $\mathrm{N}$ are shown to demonstrate how the weights $w$ of molecular data differ from patient to patient. This is indicated by variable submersions of the informational concentric cones into the rugby ball. Each single informational cone represents one of the six biomarkers that we measured. For further details see text.

symmetry, representing the 3 rotated principal components $\mathrm{c}_{1}, \mathrm{c}_{3}$ and $\mathrm{c}_{5}$ (dotted component axes in Fig. 4). Thus, VrPCA allows us to reduce our six experimental variables (five p73 isoforms plus p53 status) to only 3 principal components $\left(\mathrm{c}_{1}\right.$, $\mathrm{c}_{3}, \mathrm{c}_{5}$; see below). Together, these 3 components are necessary and sufficient to characterize the common, disease-related information for all patients. For each individual patient, these 3 components are then weighted individually so that each patient is defined by 3 specific coefficients called principal component weights $\mathrm{w}_{1}, \mathrm{w}_{3}, \mathrm{w}_{5}$ (black arrows in Fig. 4, generated by VrPCA analysis, see below). In the VrPCA algorithm, the weights $w$ are determined from the transposed matrix of Eigenvectors of the correlation matrix of profiles in Fig. 5A. These coefficients determine the weight of their respective components and thus the unique volume and form of the informational 'rugby ball' for each patient. Within the overall sphere, our molecular dataset provides a limited fraction of the total information, represented by a set of submerged concentric cones oriented towards the center of the sphere. Each single informational cone represents one of the six biomarkers that we measured. We also note that the relative proportion of individual vs. disease-specific experimental information differs from patient to patient. This is depicted by the fact that the disease-related portion of the cone that is submerged into the 'rugby ball' varies among patients, depending on the volume and shape of the 'rugby ball' (see overlap area between blue and rainbow colors in Fig. 4).

The scheme in Fig. 4 also demonstrates the requirements for further data processing: first we need to find the proper combination of experimental data that reflects the diseaserelated information. Next, the 'weights' of these diseaserelevant contributions need to be determined individually for 

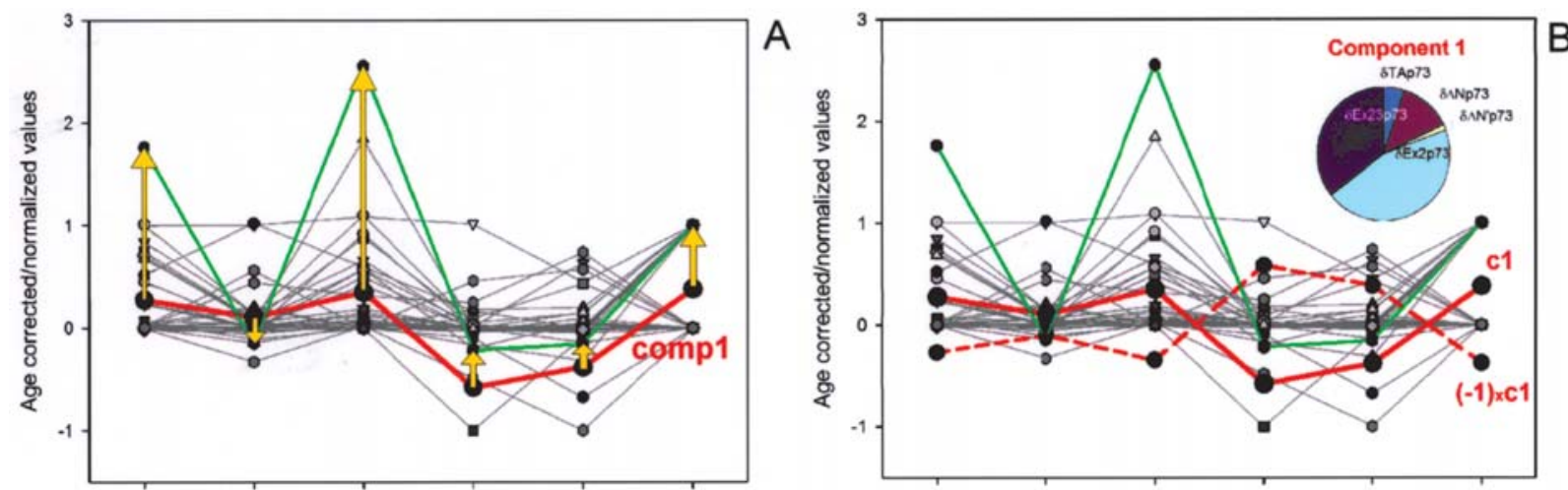

$\begin{array}{llllll}\delta T A p 73 & \delta \Delta N p 73 & \delta \Delta N ' p 73 & \delta E x 23 & \delta E x 2 & \text { p53 status }\end{array}$
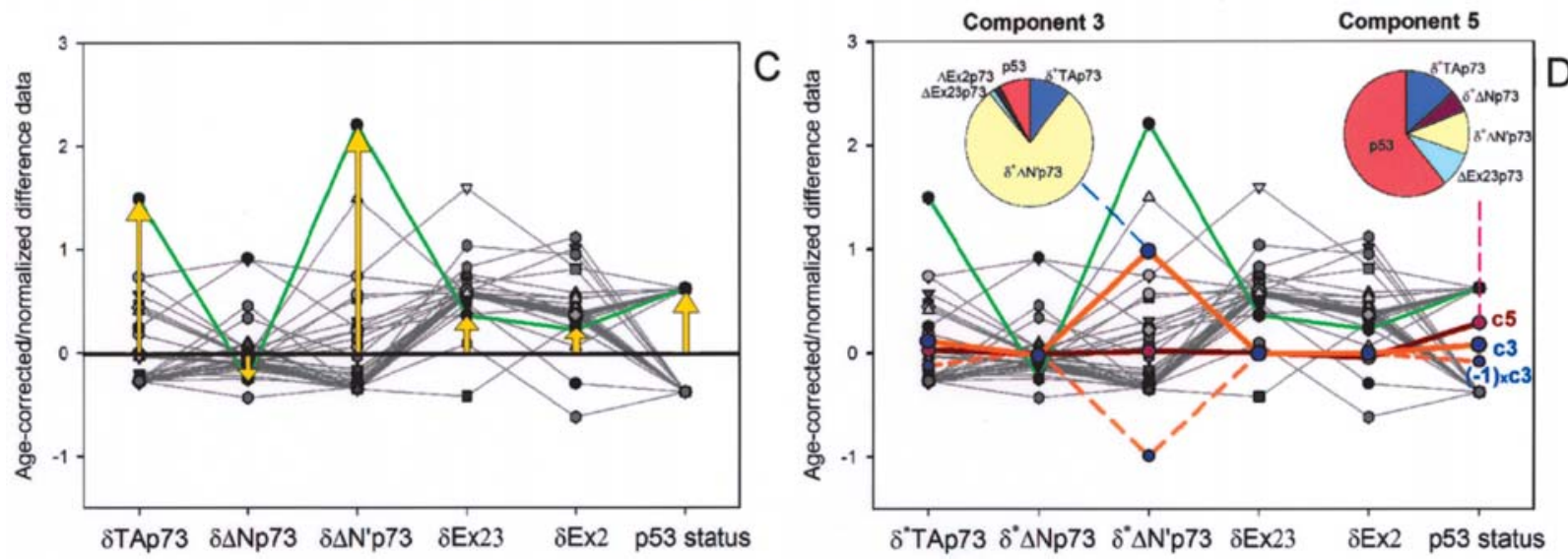

Figure 5. Generation of principal components by PCA. As a very simplified, but helpful analogy, components are essentially found by serial subtractions of a consensus profile from individual patient profiles. (A) shows the complete set of expression profiles for all 35 patients. The first component (bold red line) is derived by identifying the 'consensus variance pattern' of the $\delta$ values for all p73 isoforms and the p53 status. The profile of one patient (green line) is selected as an example to demonstrate the principle of generating the remaining components. Once the first component is determined by PCA, the red component 1 is subtracted from all individual profiles (e.g. yellow arrows for the green patient). These calculated differences are then plotted as new curves (C) that allow to generate a 2 nd order common variance pattern, i.e. component 2 (not shown). Thus, component 2 represents the common part of the largest deviations from the expression profile of an 'average patient' characterized by component 1 . This process is then systematically repeated, i.e. the second component is subtracted from the profiles in (C) and third-order deviation profiles are determined. Component 3 describes the common trend in this higher-level adjustment etc. This process is repeated until the residual profiles decrease to zero. (D) shows components 3 and 5, providing information on how the experimental variables contribute to their PCA-determined combination (see pie diagram insets in B and D), and resulting in the components' particular shape (bold lines). As the coefficients $w 1-w 5$ might be positive or negative, we also show the sign-inversed shapes of calculated components for clarity (dotted red and orange lines in B and D).

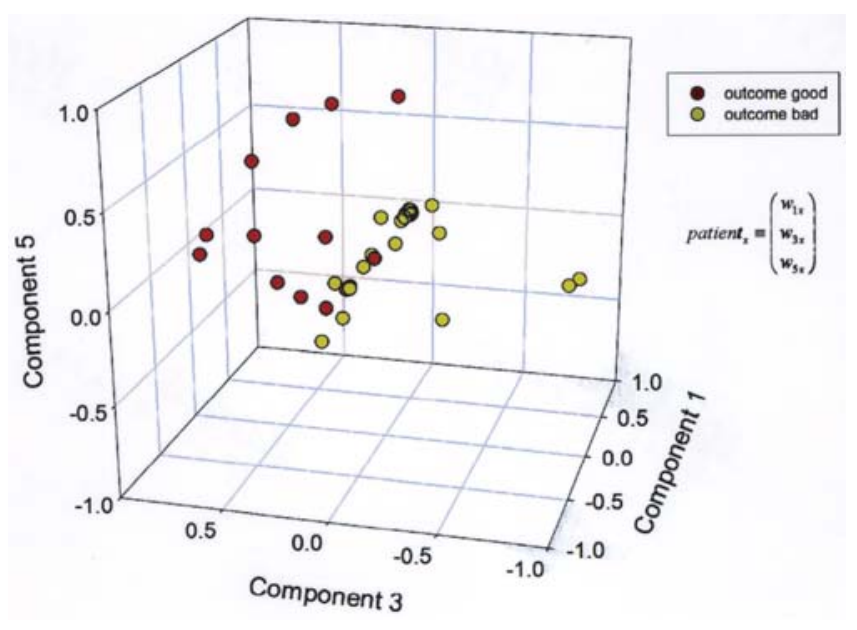

Figure 6. The PCA/Varimax components 1,3 and 5 are capable of separating the 35 patients into two prognostic subgroups, i.e. good outcome vs. poor outcome. In this 3-dimensional biomarker space, each patient is represented by a point $\left(\mathrm{x}=\mathrm{w}_{1}\right.$ on $\mathrm{C}_{1}, \mathrm{y}=\mathrm{w}_{3}$ on $\mathrm{C}_{3}, \mathrm{z}=\mathrm{w}_{5}$ on $\left.\mathrm{C}_{5}\right)$. Note that the seemingly overlapping position of some patients in this particular view would resolve upon rotation of the $3 \mathrm{D}$ space. each patient. For this purpose, we characterize each patient by an experimental profile that is individually shaped (Fig. 5). This profile has the 6 input variables, ordered systematically on the $\mathrm{X}$-axis (Fig. 5). We then use Varimax rotated principal component analysis to decompose the shapes of these profiles into independent components. In a very simplified, but helpful analogy, components are basically found by serial subtractions of a consensus profile from individual patient profiles. (For further explanation see Fig. 5). Thus, the main purpose of PCA is to identify directions with large variances and to reduce dimensionality. For a given patient, we can reconstruct the original experimental data by multiplying the 5 principal components $\left(\mathrm{c}_{1}, \mathrm{c}_{2}, \mathrm{c}_{3}, \mathrm{c}_{4}, \mathrm{c}_{5}\right)$ with the patient's specific coefficients $\mathrm{w}_{\mathrm{k}}\left(\mathrm{w}_{1}, \mathrm{w}_{2}, \mathrm{w}_{3}, \mathrm{w}_{4}, \mathrm{w}_{5}\right)$ and summing up the products. This is proof that we are not losing any information by applying the PCA/Varimax algorithm. We are only redistributing the experimental input data in such a way that allows us to identify the disease-specific part within the original information, as well as the relative contribution of our 6 experimental variables to the final prediction of clinical outcome. 
Table IV. Resulting predictions made by the SVM based on Fig. 6.

\begin{tabular}{lrrr}
\hline \multirow{2}{*}{ Prediction of prognosis } & \multicolumn{2}{c}{ Real prognosis } & \multirow{2}{*}{ Sum } \\
& Good & Poor & \\
\hline 'Good' & 14 & 5 & 19 \\
'Poor' & 0 & 16 & 16 \\
Sum & 14 & 21 & 35 \\
\hline
\end{tabular}

VrPCA combines the 6 input variables into 5 principal components. Note that each of these 5 components contains all 6 variables, albeit weighted to different degrees, since the PCA method is based upon the correlation matrix. The $\mathrm{w}_{\mathrm{k}}$ coefficients are therefore normalized before entering the SVM. This normalization is reflected by an additional condition for the $\mathrm{w}_{\mathrm{k}}$ values for each patient, namely $\sum_{k=1}^{6} w_{k}^{2}=1$. This additional equation automatically reduces the dimension of the problem from 6 to 5 , because one can calculate the sixth $\mathrm{w}_{\mathrm{k}}$ value by knowing the five others and by using the above normalization condition. This is also clearly shown by the fact that the 6th component coefficients, as calculated by the VrPCA, are negligibly small (in the order of $10^{-9}$ and less) and are non-zero only due to the unavoidable rounding error of the computational algorithm, which is due to the finite representation of real numbers in any computer. Thus, the full set of coefficients that completely represent the data is used, without selection bias and the sum of the first five eigenvalues is 6 . In our data set, we also found a strong linear relation between $\delta^{*} \mathrm{TAp} 73$ and $\delta^{*} \Delta \mathrm{N}^{\prime} \mathrm{p} 73$, which we had already observed in our previous study (29), and between $\delta^{*} \Delta \mathrm{Np} 73$ and $\delta^{*} \mathrm{Ex} 2 / 3 \mathrm{p} 73$.

Next, we subject the obtained PCA solution to Varimax rotation. The final coefficients discussed here are generated by Varimax rotation of the PCA solution. Varimax rotation is one of the possible methods designed to minimize the problems of PCA components for data interpretation that stem from its formal mathematic derivation. Varimax rotation removes the artificial orthogonality condition required for PCA components and maximizes the co-linearity of the rotated components with selected vectors of real data. The algorithm is derived by that condition. It therefore takes the PCA solution and transforms the orthogonal PCA coordinate system into an alternative, non-orthogonal coordinate system. Axes in this new rotated non-orthogonal system are maximally co-linear with selected columns of experimental data. This therefore provides not a perfect, but for a given case the optimal basis for correct interpretation of the rotated components. Component compositions are shown by pie diagrams in Fig. 5. Component $\mathrm{c}_{1}$ mainly describes disease-correlated $\delta \mathrm{Ex} 2$ and $\delta \mathrm{Ex} 2 / 3 \mathrm{p} 73$ changes. However, $\mathrm{c}_{1}$ only has a high weighting coefficient for those patients who showed a significant tumor-associated up- or down-regulation of these isoforms in their tumors. Component $\mathrm{c}_{3}$ predominantly characterizes changes in patients' $\delta^{*} \Delta \mathrm{N}^{\prime} \mathrm{p} 73$, whereas $\mathrm{c}_{5}$ is mostly related to the mutation status of $\mathrm{p} 53$.
Table V. Average errors for the individual rotated components.

\begin{tabular}{ccccc}
\hline $\mathrm{w}_{1}$ & $\mathrm{w}_{2}$ & $\mathrm{w}_{3}$ & $\mathrm{w}_{4}$ & $\mathrm{w}_{5}$ \\
\hline 0.004594 & 0.007215 & 0.00672 & 0.013689 & 0.007199 \\
\hline
\end{tabular}

We next addressed the stability of data pre-processing by VrPCA. To this end, we performed 35 independent rounds of VrPCA processing of input data for subsets of 34 patients each, with a different single patient systematically left out in each round. The resulting weights $\mathrm{w}_{1, \text { oneout }}-\mathrm{w}_{5, \text { oneout }}$ for rotated components were collected. We then calculated the differences of these coefficients from the coefficients $\mathrm{w}_{1, \text { full }}-\mathrm{W}_{5, \text { full }}$. This calculation was performed for each patient. Altogether, this resulted in collecting 6480 differences

$$
\Delta w_{k, i}=\sqrt{\left(w_{k, i, \text { one out }}-w_{k, i, \text { full }}\right)^{2}} .
$$

Fig. 7 shows the distribution of the differences $\Delta w_{k, i}$ for our data set. Since our coefficients are normalized, their values fall between -1.0 and +1.0 . As evident from the figure, $90 \%$ of the coefficient variations lie within $2 \%$ of the values we used in the testing. Table $\mathrm{V}$ summarizes the average errors for the individual rotated components. They were calculated by first averaging $\Delta w_{k, i}$ for each component and each patient and then by averaging them over all patients in the set.

Towards our final goal of a prognostic model, we next used the robust algorithm of support vector machine (SVM) (40) that was trained to predict outcomes from the calculated patient-specific coefficients $\mathrm{w}_{1}-\mathrm{w}_{5}$. The definitions were outcome I, no evidence of disease; outcome II, recurrence or alive with disease; outcome III, death of disease. However, not all of these weighting coefficients are required for prediction. On the contrary, some of them have to be eliminated because they predominantly represent the individual, non-disease related variability of expression profiles (data not shown). To choose the optimal combination of weights, we applied an exhaustive feature selection approach. First, all five coefficients were used individually as input into SVM. All models were subjected to leave-one-out testing of the robustness of their predictive capability. Then we tested all possible pair wise combinations of five coefficients, followed by all possible combinations of three, four and five coefficients. In this empirical testing, only one combination of weights, the triplet of components 1,3 and 5, had the power to predict clinical outcome in our cohort (Fig. 6). In the conceptual model of Fig. 4, this means that only principal components 1,3 and 5 represent the information that is disease-related and thus are needed to predict outcome. In contrast, components 2 and 4 largely contain the individual non-disease related part of our experimental data.

Next, it was important to examine the impact of the observed variations on the predictions generated by the support vector machine equations (SVME). This was tested by analyzing the distances $\mathrm{D}_{\mathrm{i}}(\mathrm{X}-\mathrm{Y})$ from the separation planes (calculated by SVME) for variations of component weights $\mathrm{W}_{\mathrm{i}}$ within the limits of the $\pm \Delta w_{k i}$. The impact of data pre- 


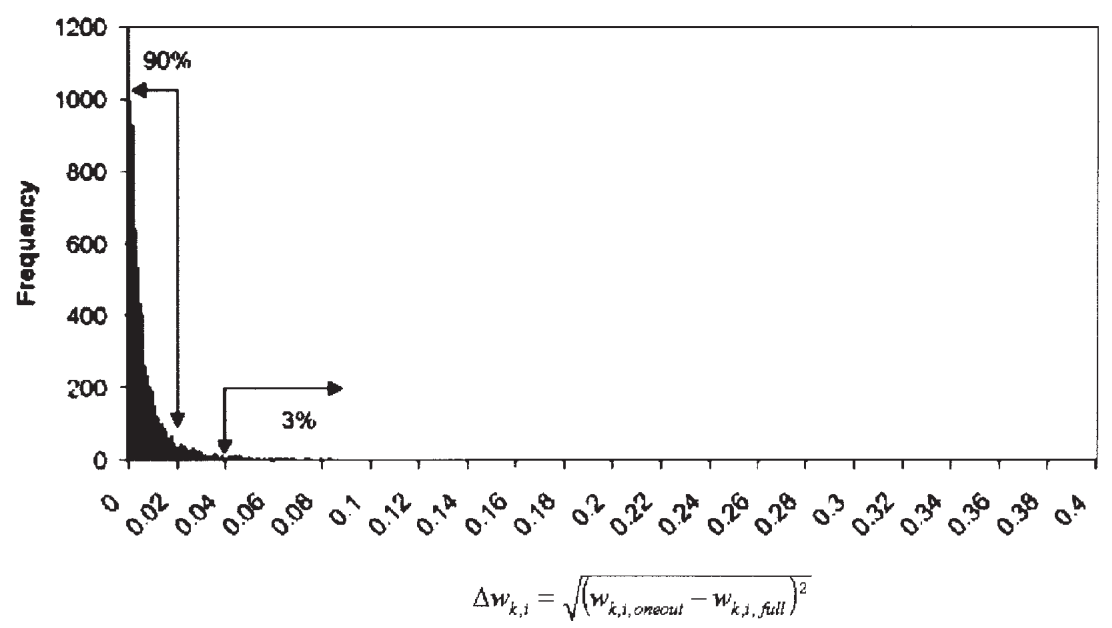

Figure 7. A high stability of data pre-processing by VrPCA rotation is reflected by the narrow distribution of coefficient 1-5 variations for 35 one-out $\mathrm{VrPCA}$ tests.

processing by VrPCA was quantified by a shift of the separating plane as defined by SVME. Throughout the space of possible variations of $\pm \Delta w_{k i}$, this resulted in a parallel shift of the separating plane. The shift was maximal for errors with alternating signs of correction $\pm \Delta w_{k i}$, and was about $1 / 3$ of the maximum for errors with the same signs of $\pm \Delta w_{k i}$. Thus, for the worst case scenario of $\pm\left|\Delta w_{k i}\right| \sim 0.04$ with alternating signs (Table V), the separating planes shifted by maximally 0.2 in the z-direction. As the range of distances within all possible values of $\mathrm{Wij}$ coefficients is 6.0 , this shift represents a relative error of $3.3 \%$. Since it scales linearly with $\pm \Delta w_{k i}$, it follows that for $90 \%$ of our test cases, the maximum relative shift along the z-axis of the decision SVM planes is only $1.7 \%$. Moreover, this will affect only the borderline cases that lie along the decision planes and are problematic anyway. On the other hand, the very essence of the SVM algorithm is that it maximizes the distances of patient coordinates from these decision planes. Therefore, by choosing this algorithm, we minimized the risk of these problems derived from possible preprocessing errors. Moreover, our prediction is based upon the sign of the distance only, not upon the sign plus numerical value, which de facto minimizes the impact of the $\mathrm{w}_{\mathrm{k}}$ variation. This is why we can only discuss the shift of the plane as relevant, because only in this small region surrounding the intersection between decision planes and coefficient planes, can the shift of the plane result in a sign change and thus in different outcome.

Fig. 6 shows the cohort of 35 patients placed into a 3dimensional space whose axes are the components $\mathrm{c}_{1}, \mathrm{c}_{3}$ and $\mathrm{c}_{5}$. When each patient is coded by outcome according to 'good' (free of disease, group I) and 'bad' (alive with disease and dead of disease, groups II and III), these two new groups are separated by a non-planar surface. The goal of the SVM algorithm is to find the optimal plane that separates good from bad outcome. The actual curved separating surface we observed was therefore approximated by a combination of 3 planes separating group I from III, group I from II and group II from III. In detail, we trained SVM to find a single plane that optimally separates group I from II, another plane that optimally separates group I from III and finally the plane that optimally separates group II from III. 'Optimal' means that the distances of all points from the separating plane are maximal. This minimizes the risk of false classification. The result of the training are the separating plane positions and equations (see formulae below), which calculate the distance $\mathrm{D}_{\mathrm{i}}$ of each of the $i=1 . .35$ patient points $\left[\mathrm{w}_{1 \mathrm{i}}, \mathrm{w}_{3 \mathrm{i}}, \mathrm{w}_{5 \mathrm{i}}\right]$ from all three planes.

$$
\begin{aligned}
& \mathrm{D}_{\mathrm{i}}(\mathrm{I}-\mathrm{II})=1.9026 \mathrm{w}_{1 \mathrm{i}}-0.9836 \mathrm{w}_{3 \mathrm{i}}-0.6841 \mathrm{w}_{5 \mathrm{i}}-0.9343 \\
& \mathrm{D}_{\mathrm{i}}(\mathrm{I}-\mathrm{III})=2.4284 \mathrm{w}_{1 \mathrm{i}}-2.029 \mathrm{w}_{3 \mathrm{i}}-1.1743 \mathrm{w}_{5 \mathrm{i}}+0.0917 \\
& \mathrm{D}_{\mathrm{i}}(\mathrm{II}-\mathrm{III})=0.0121 \mathrm{w}_{1 \mathrm{i}}-0.3424 \mathrm{w}_{3 \mathrm{i}}-0.2288 \mathrm{w}_{5 \mathrm{i}}+1.2561
\end{aligned}
$$

The distance to the separating plane is defined as negative for the outcome classification lying to the left of the separating plane (e.g. I in the separating 'I-III' plane) and positive for the outcome classification lying to the right of the plane (e.g. III in the separating 'I-III' plane). Thus, for a given patient, if $\mathrm{D}(\mathrm{I}-\mathrm{III})<0$, we predict outcome I (no evidence of disease), if $\mathrm{D}(\mathrm{I}-\mathrm{III})>0$, we predict outcome III (death of disease), etc. For each patient, we have three $\mathrm{D}$-values. The correct prediction is further defined as the majority of classification results. For example, for a patient with a [I, I, III] result, we predict outcome I, because I is the majority of the outcome classifications.

To test the robustness of our prognostic model, we performed leave-one-out testing. This means that one patient was taken out of our cohort of 35 patients and a prediction for this patient was made based upon the remaining 34 patients as training set. This procedure was repeated for each patient, thus 35 times. Using this method, the SVM algorithm predicted all 14 cases of outcome I correctly, furthermore 11 of the 13 cases of outcome III were accurately classified, whereas only 2 cases were falsely classified as outcome I. SVM was not able to predict the 8 cases of outcome II. Instead, it classified 3 cases incorrectly as outcome I and 5 cases as outcome III. However, after we pooled patients with outcome II and III to the 'bad' prognosis group, as opposed to the 'good' prognosis group of outcome I, $86 \%$ of cases were correctly classified [30 out of 35 ; all 14 patients $(100 \%)$ of the good prognosis 

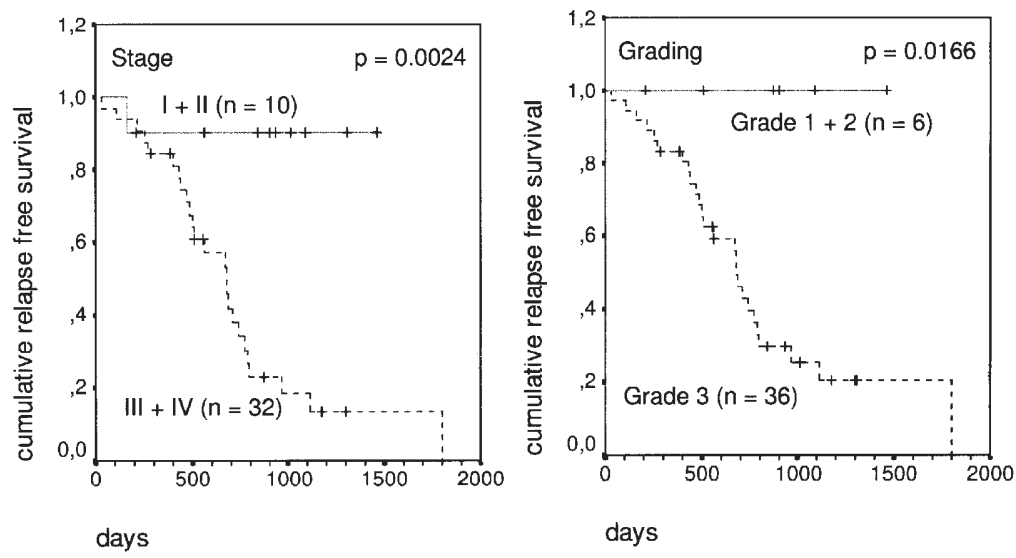

Figure 8. Kaplan-Meier survival curves in 42 gynecological cancers according to tumor stage and histological grading. Patients with stage I/II tumors, or patients with grade $1 / 2$ have a significant longer relapse-free survival than patients with stage III/IV tumors or grade 3 tumors. Censored patients are marked with a cross.

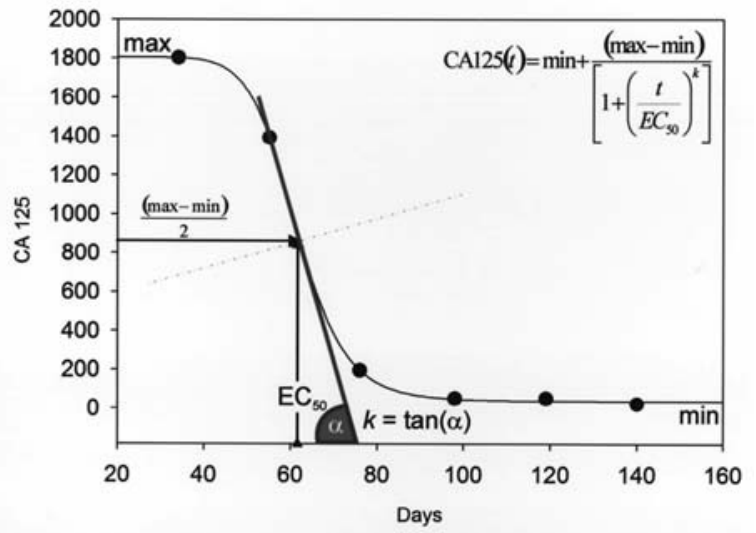

Figure 9. The CA125 time course of each patient can be characterized by 4 points: the maximum value after surgery but before the first chemotherapy cycle, the slope defining the drop per time unit (i.e. the rate), the $\mathrm{EC}_{50}$ and the minimum stable level after finishing the postoperative chemotherapy regimen.

group as well as 16 of the 21 patients $(76 \%)$ of the bad prognosis group were correctly identified] (Table IV). Thus, the specificity to detect patients with a good prognosis was $100 \%$ and the sensitivity to identify patients with bad prognosis was $76 \%$. The negative predictive value, i.e. the probability that a patient with a predicted good prognosis in fact really had a favorable outcome was 74\% (14/19), whereas the positive predictive value indicating the probability with which a patient, for whom a bad outcome was predicted, really experienced recurrence or death was $100 \%$ (16/16) (Table IV). On the other hand, using p73 $\delta$ values as input, we tested a broad range of other algorithms (raw, non-VrPCA treated data, even with and without age correction of expression levels; see below) for predicting the known clinical outcome of our cohort. These included Bayesian classifiers, back-propagation neural network, clustering methods and support vector machine algorithms from the Weka package (39). However, all these methods failed to achieve predictive significance, whether $\delta$ values were age-corrected or not. Moreover, reducing input by systematically selecting only data subsets also failed to improve the predictive significance of each algorithm.
A poor drop of CA125 correlates with a high $\triangle T A p 73 / T A p 73$ ratio. For 17 of our ovarian cancer patients, CA125 serum levels, sampled before each chemotherapy cycle were available. This allowed us to create individual CA125 curves (an example is shown in Fig. 9). When plotted over time, CA125 values could be fitted into a 4-parameter sigmoidal function (Sigmaplot v. 7.0 package, SPSS Inc.). For correlation analyses, the ratio of $\delta^{*} \Delta \mathrm{TAp} 73$ (i.e. age corrected $\delta^{*} \Delta \mathrm{Np} 73$ plus $\delta^{*} \Delta \mathrm{N}^{\prime} \mathrm{p} 73$ ) over $\delta^{*} \mathrm{TAp} 73$ was calculated for each tumornormal matched pair. Patients were then separated into group I with a ratio smaller than the median and group II with a $\delta^{*} \Delta \mathrm{TA} / \delta^{*} \mathrm{TA}$ ratio equal to or higher than the median.

$\mathrm{EC}_{50}$ is defined as the time required for CA125 to drop to $50 \%$ of its initial level. A low $\mathrm{EC}_{50}$ is an established prognostic parameter for chemoresponsiveness and favorable outcome in ovarian cancer (41-43). We could confirm this correlation, thus again validating our patient dataset. In our cohort, patients with an $\mathrm{EC}_{50}$ lower than the median (51 days) had a longer relapse-free (median 1109 vs. 443 days; $p=0.0045$ ) and overall survival (median 1109 vs. 677 days; $\mathrm{p}=0.0499$ ) than patients with an $\mathrm{EC}_{50}$ equal to or higher than the median (Fig. 10). Another important value characterizing the CA125 profile is the slope (i.e. the decrease of CA125 over time), which is much less dependent on the starting level than the $\mathrm{EC}_{50}$ parameter. Thus, a steep slope (i.e. a rapidly falling CA125) mainly reflects the effect of chemotherapy and hence also serves as a parameter to assess chemoresponsiveness. To this end, we looked for a correlation between the CA125 slope and p73 levels in our cohort. We found a significant association between up-regulated $\Delta \mathrm{TAp} 73$ in tumors $(\Delta \mathrm{Np} 73$ plus $\left.\Delta \mathrm{N}^{\prime} \mathrm{p} 73\right)$ and a reduced $\mathrm{CA} 125$ slope, indicating a poor response to chemotherapy (Fig. 11). Patients with an agecorrected $\delta^{*} \Delta \mathrm{TAp} 73 / \delta^{*} \mathrm{TAp} 73$ ratio higher than the median showed a significantly lower CA125 slope in the MannWhitney test (mean 3.68 vs. 14.66; $\mathrm{p}=0.027$ ). However, when the same $\Delta \mathrm{TA} / \mathrm{TA}$ ratio was calculated for normalized but not age-corrected $\delta$ values, a correlation was no longer detectable. This again emphasizes the importance of age correction for raw $\delta$ values for $\mathrm{p} 73$ expression.

Since drug induced tumor cytotoxicity is partly mediated by functional, pro-apoptotic TAp73 and p53, an up-regulation 


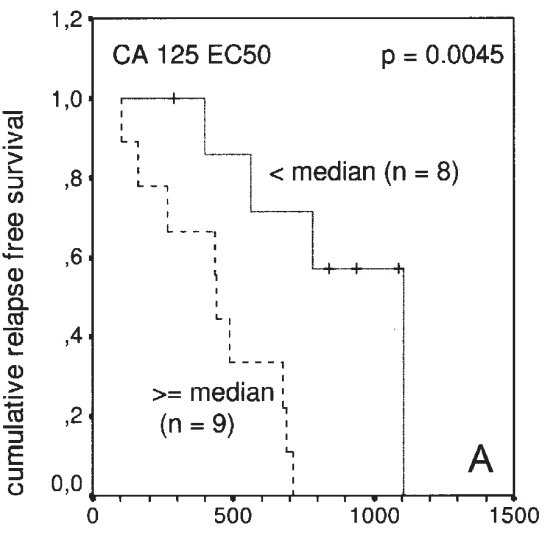

days

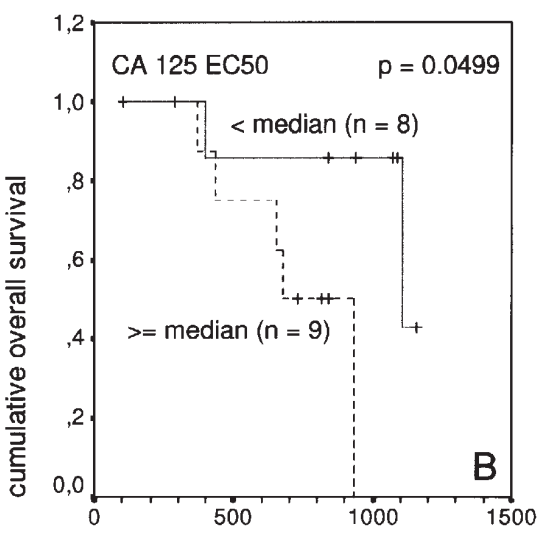

days

Figure 10. Kaplan-Meier relapse-free and overall survival curves for patients with a high $\mathrm{EC}_{50}$ value for $\mathrm{CA} 125$ compared to patients with a low value.

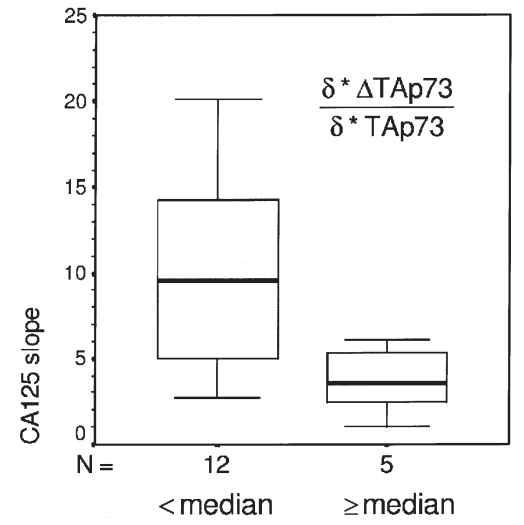

Figure 11. Boxplot comparing the CA125 slope between patients with a high $\Delta \mathrm{TA} / \mathrm{TA}$ ratio and patients with a low $\Delta \mathrm{TA} / \mathrm{TA}$ ratio.

of $\Delta \mathrm{TAp} 73$ in tumors, which inhibits TAp73 and p53, might increase drug resistance of those tumors and therefore result in worse responsiveness to chemotherapy. In fact, TAp73 is induced by a variety of chemotherapeutic agents such as cisplatin, taxol, doxorubicin and etoposide in different cancer cell lines $(2,44)$. Of note, blocking p73 function, either with dominant negative $\Delta \mathrm{Np} 73$, or with siRNA against TAp73 led to chemoresistance, irrespective of p53 status $(2,44)$. Taken together, the significant correlation between high $\delta^{*} \Delta \mathrm{TAp} 73 /$ $\delta^{*}$ TAp73 expression levels and a reduced CA125 slope indicates that up-regulation of $\triangle \mathrm{TAp} 73$ isoforms in tumors might be involved in impairing the efficacy of apoptosis inducing anti-cancer drugs.

\section{Discussion}

In the current work, we determined expression levels of all N-terminal p73 isoforms by real-time RT-PCR in a cohort of 35 gynecological cancers. Of note, this is the first such study that uses matched tumor/normal tissue comparisons, which allows to calculate up-regulation of all isoforms for each patient. Previous studies relied on unmatched pooled normal tissues from unrelated individuals for comparisons. Consistent with previous studies, we found that TAp $73, \Delta \mathrm{Np} 73$ and
$\Delta \mathrm{N}^{\prime} \mathrm{p} 73$ were significantly up-regulated in tumors compared to matched normal tissues. Surprisingly, however, the range of overexpression of TA, $\Delta \mathrm{N}$ and $\Delta \mathrm{N}^{\prime} \mathrm{p} 73$ in tumors was agedependent, with the highest values present in the youngest age group. Such age dependence of p73 isoform expression had not been previously reported but appears to be important, since this isoform-specific age correction turned out to be crucial to reveal significance in subsequent survival correlations. Thus, we propose that age dependence should be taken into account in future prognostic $\mathrm{p} 73$ studies. $\Delta \mathrm{N}$ forms of p73 have been shown to act in a dominant-negative manner and hence their up-regulation in human tumors could well be oncogenic. The significance of the often observed co-upregulation of TAp73 remains unclear but might be a side effect of P1 promoter activation which generates 3 of the 4 truncated isoforms.

There is mounting evidence for a prognostic significance of p73 expression in human cancers. Initially, a study in 193 hepatocellular carcinomas found that immunohistochemical detectability of p73 was an independent prognostic factor for poor outcome (28). Likewise, in colorectal and ovarian carcinomas, immunohistochemical p73 overexpression independently predicted poor prognosis $(26,27)$. Also, total p 73 mRNA overexpression in a subgroup of 70 breast cancers correlated with poor clinical behaviour (25). However, all these studies analysed total p73 expression and did not discriminate between different N-terminal p73 isoforms, despite their different, even opposing functions.

Taking into account the opposing roles of TAp73 and $\Delta \mathrm{TAp} 73$, a few studies then investigated the prognostic role of $\Delta \mathrm{Np} 73$ as a potential predictor of poor outcome in cancer. Until now, this analysis was restricted to classical KaplanMeier survival correlations using isolated isoforms or simple ratios of isoforms. Of note, this approach required relatively large cohorts to detect a correlation. $\Delta \mathrm{Np} 73$ up-regulation was found to be an independent predictor of poor outcome in 51 neuroblastomas and in 132 lung cancers $(23,24)$. In two prior studies to the one presented here, we had also analysed all N-terminal p73 isoforms. In the initial cohort of 100 ovarian cancers, we found a non-significant trend for better overall survival in patients with low expression of $\Delta N^{\prime} p 73 / \Delta N p 73$ compared to patients with high expression (29). Only a sub- 
sequent study, extended to 122 ovarian cancers, did reveal a significant correlation. High expression levels of dominantnegative $\Delta \mathrm{Np} 73 / \Delta \mathrm{N}$ 'p 73 significantly correlated with chemotherapeutic failure $(\mathrm{p}=0.05)$ in all patients and correlated with worse recurrence-free and overall survival in those patients with p53 mutant cancers ( $\mathrm{p}=0.048$ and 0.005 , respectively) (30).

However, within our cohort of 35 patients, we were at first unable to detect a significant simple correlation between one experimental parameter (such as expression of individual isoforms like $\Delta \mathrm{N}^{\prime} \mathrm{p} 73 / \Delta \mathrm{Np} 73$ ) or ratios thereof and clinical outcome. Therefore, we applied the combinatorial approach of principal component analysis to comprehensively capture all molecular variables measured. Selected principal components were then used to train a support vector machine algorithm to predict clinical outcome. The reliability of PCA and SVM as powerful statistical tools in predicting clinical outcome of tumors has been previously demonstrated in microarraybased gene expression profiling (45). In a study on neuroblastoma patients, Wei et al used PCA to reduce the dimensionality of the cDNA microarray data initially containing 37,920 clones to the top 10 principal components. These top components were then used as input into an artificial neural network. Subsequent prognosis prediction, based on one-out testing, achieved $88 \%$ accuracy in discriminating between neuroblastoma patients with good vs. poor prognosis (45). Likewise, Williams et al analyzed 27 samples of Wilms tumor by cDNA microarrays and applied a SVM algorithm with leave-one-out testing to identify those genes whose expression is predictive of relapse (46). Thus, we applied a combination of established, reliable statistical methods for our predictive model. Overall, we used 6 molecular variables (p73 isoform levels plus p53 status) as input into the PCA/Varimax algorithm to test whether they could serve as combinatorial biomarkers for predicting outcome in this cohort. Of the resulting 5 principal components for each patient, only 3 weighted components $\left(\mathrm{c}_{1}, \mathrm{c}_{3}, \mathrm{c}_{5}\right)$, representing the prognostically relevant part of our experimental data set, were used to train the SVM on our cohort of 35 patients. This allowed us to correctly predict the clinical outcome of 30 out of the 35 patients (leave-one-out testing).

This model allows a context-related view of the prognostic value of an individual variable. This is important because we show that the predictive meaning of any one particular biomarker, e.g. the expression level of a specific p73 isoform, might change depending on the context of the other 5 molecular variables. On the one hand, the principal components of all patients share the same composition, which reflects the disease-related, common variance of experimental variables in our cohort. On the other hand, these components differ in their respective weights from patient to patient, thus individualizing the prediction. Hence, for some patients, component 3 , of which $\delta^{*} \Delta \mathrm{N}^{\prime}$ is the main contributor, might have the utmost weight in predicting survival, while the other components are much less important. However, for other patients, component 3 might only be of minor importance compared to the other components. Of note, this does not mean that any of the 6 molecular variables is completely unnecessary for survival prediction of a given patient, since all principal components contain all 6 variables, only in different proportions.
This method also considers the individual patient's response to the disease according to the particular combination of genetic and environmental factors. We do not exclude the possibility that other oncogenic factors, which we did not analyse here also contributed to the disease, but we find that p73 isoform and p53 variables are sufficient for prognostic purposes. Thus, this method is open to future expansions that incorporate other molecular markers.

Our model draws its statistical power from exploiting the predictive information contained in combining all measured parameters, thus creating a unique experimental profile for each patient. In contrast to a single parameter, this combinatorial concept might also better reflect the frequent co-up-regulation of p73 isoforms and the complex network of interrelationships between different p53 family members. In sum, we have shown that in this cohort of cancer patients clinical outcome did not correlate with a single molecular p73/p53 parameter. However, with the proper selection of mathematical tools, we were able to integrate p73 isoform expression profiles and p53 status into useful prognostic biomarkers with the power to differentiate patients with good vs. poor prognosis. A prognostic classification based on this integrative system might be of use in the clinic to help identify patients who might benefit from more aggressive adjuvant chemotherapy.

\section{Acknowledgements}

This study was supported by grant RO1 CA93853 from the National Cancer Institute to Ute M. Moll. Nicole Concin was supported by the Austrian FWF (Fonds zur Förderung der wissenschaftlichen Forschung) and by the Erwin-Schroedinger Stiftung.

\section{References}

1. Agami R, Blandino G, Oren M and Shaul Y: Interaction of $\mathrm{c}-\mathrm{Abl}$ and p73alpha and their collaboration to induce apoptosis. Nature 399: 809-813, 1999.

2. Bergamaschi D, Gasco M, Hiller L, Sullivan A, Syed N, Trigiante G, Yulug I, Merlano M, Numico G, Comino A, Attard M, Reelfs O, Gusterson B, Bell AK, Heath V, Tavassoli M, Farrell PJ, Smith P, Lu X and Crook T: p53 polymorphism influences response in cancer chemotherapy via modulation of p73-dependent apoptosis. Cancer Cell 3: 387-402, 2003.

3. Gong JG, Costanzo A, Yang HQ, Melino G, Kaelin WG Jr, Levrero $M$ and Wang JY: The tyrosine kinase c-Abl regulates p73 in apoptotic response to cisplatin-induced DNA damage. Nature 399: 806-809, 1999.

4. Irwin M, Marin MC, Phillips AC, Seelan RS, Smith DI, Liu W, Flores ER, Tsai KY, Jacks T, Vousden KH and Kaelin WG Jr: Role for the p53 homologue p73 in E2F-1-induced apoptosis. Nature 407: 645-648, 2000

5. Lissy NA, Davis PK, Irwin M, Kaelin WG and Dowdy SF: A common E2F-1 and p73 pathway mediates cell death induced by TCR activation. Nature 407: 642-645, 2000.

6. Stiewe T and Putzer BM: Role of the p53-homologue p73 in E2F1-induced apoptosis. Nat Genet 26: 464-469, 2000.

7. Zaika A, Irwin M, Sansome C and Moll UM: Oncogenes induce and activate endogenous p73 protein. J Biol Chem 276: 11310-11316, 2001

8. Yang A, Walker N, Bronson R, Kaghad M, Oosterwegel M, Bonnin J, Vagner C, Bonnet H, Dikkes P, Sharpe A, McKeon F and Caput D: p73-deficient mice have neurological, pheromonal and inflammatory defects but lack spontaneous tumours. Nature 404: 99-103, 2000.

9. Flores ER, Sengupta S, Miller JB, Newman JJ, Bronson R, Crowley D, Yang A, McKeon F and Jacks T: Tumor predisposition in mice mutant for p63 and p73: evidence for broader tumor suppressor functions for the p53 family. Cancer Cell 7: 363-373, 2005. 
10. Melino G, De Laurenzi V and Vousden KH: p73: Friend or foe in tumorigenesis. Nat Rev Cancer 2: 605-615, 2002

11. Grob TJ, Novak U, Maisse C, Barcaroli D, Luthi AU, Pirnia F, Hugli B, Graber HU, De Laurenzi V, Fey MF, Melino G and Tobler A: Human delta Np73 regulates a dominant negative feedback loop for TAp73 and p53. Cell Death Differ 8: 1213-1223, 2001.

12. Pozniak CD, Radinovic S, Yang A, McKeon F, Kaplan DR and Miller FD: An anti-apoptotic role for the p53 family member, p73, during developmental neuron death. Science 289: 304-306, 2000.

13. Stiewe T, Theseling CC and Putzer BM: Transactivation-deficient Delta TA-p73 inhibits p53 by direct competition for DNA binding: implications for tumorigenesis. J Biol Chem 277: 14177-14185, 2002.

14. Ishimoto O, Kawahara C, Enjo K, Obinata M, Nukiwa T and Ikawa S: Possible oncogenic potential of DeltaNp73: a newly identified isoform of human p73. Cancer Res 62: 636-641, 2002.

15. Kaghad M, Bonnet H, Yang A, Creancier L, Biscan JC, Valent A, Minty A, Chalon P, Lelias JM, Dumont X, Ferrara P, McKeon F and Caput D: Monoallelically expressed gene related to p53 at $1 \mathrm{p} 36$, a region frequently deleted in neuroblastoma and other human cancers. Cell 90: 809-819, 1997.

16. Stiewe T and Putzer BM: Role of p73 in malignancy: tumor suppressor or oncogene? Cell Death Differ 9: 237-245, 2002.

17. Zaika AI, Slade N, Erster SH, Sansome C, Joseph TW, Pearl M, Chalas E and Moll UM: DeltaNp73, a dominant-negative inhibitor of wild-type p53 and TAp73, is up-regulated in human tumors. J Exp Med 196: 765-780, 2002.

18. Fillippovich I, Sorokina N, Gatei M, Haupt Y, Hobson K, Moallem E, Spring K, Mould M, McGuckin MA, Lavin MF and Khanna KK: Transactivation-deficient p73alpha (p73Deltaexon2) inhibits apoptosis and competes with p53. Oncogene 20: 514-522, 2001.

19. Nakagawa T, Takahashi M, Ozaki T, Watanabe KK, Todo S, Mizuguchi H, Hayakawa T and Nakagawara A: Autoinhibitory regulation of $\mathrm{p} 73$ by Delta Np73 to modulate cell survival and death through a p73-specific target element within the Delta Np73 promoter. Mol Cell Biol 22: 2575-2585, 2002.

20. Slade N, Zaika AI, Erster S and Moll UM: DeltaNp73 stabilises TAp73 proteins but compromises their function due to inhibitory hetero-oligomer formation. Cell Death Differ 11: 357-360, 2004.

21. Petrenko O, Zaika A and Moll UM: deltaNp73 facilitates cell immortalization and cooperates with oncogenic Ras in cellular transformation in vivo. Mol Cell Biol 23: 5540-5555, 2003.

22. Stiewe T, Zimmermann S, Frilling A, Esche H and Putzer BM: Transactivation-deficient DeltaTA-p73 acts as an oncogene. Cancer Res 62: 3598-3602, 2002.

23. Casciano I, Mazzocco K, Boni L, Pagnan G, Banelli B, Allemanni G, Ponzoni M, Tonini GP and Romani M: Expression of DeltaNp73 is a molecular marker for adverse outcome in neuroblastoma patients. Cell Death Differ 9: 246-251, 2002.

24. Uramoto H, Sugio K, Oyama T, Nakata S, Ono K, Morita M, Funa $\mathrm{K}$ and Yasumoto K: Expression of deltaNp73 predicts poor prognosis in lung cancer. Clin Cancer Res 10: 6905-6911, 2004.

25. Dominguez G, Silva JM, Silva J, Garcia JM, Sanchez A, Navarro A, Gallego I, Provencio M, Espana P and Bonilla F: Wild-type p73 overexpression and high-grade malignancy in breast cancer. Breast Cancer Res Treat 66: 183-190, 2001.

26. Niyazi M, Ghazizadeh M, Konishi H, Kawanami O, Sugisaki Y and Araki T: Expression of p73 and c-Abl proteins in human ovarian carcinomas. J Nippon Med Sch 70: 234-242, 2003.

27. Sun XF: p73 overexpression is a prognostic factor in patients with colorectal adenocarcinoma. Clin Cancer Res 8: 165-170, 2002 .

28. Tannapfel A, Wasner M, Krause K, Geissler F, Katalinic A, Hauss J, Mossner J, Engeland K and Wittekind C: Expression of p73 and its relation to histopathology and prognosis in hepatocellular carcinoma. J Natl Cancer Inst 91: 1154-1158, 1999.

29. Concin N, Becker K, Slade N, Erster S, Mueller-Holzner E, Ulmer H, Daxenbichler G, Zeimet A, Zeillinger R, Marth C and Moll UM: Transdominant DeltaTAp73 isoforms are frequently up-regulated in ovarian cancer. Evidence for their role as epigenetic p53 inhibitors in vivo. Cancer Res 64: 2449-2460, 2004.
30. Concin N, Hofstetter G, Berger A, Gehmacher A, Reimer D, Watrowski R, Tong D, Schuster E, Hefler L, Heim K, MuellerHolzner E, Marth C, Moll UM, Zeimet AG and Zeillinger R: Clinical relevance of dominant-negative p73 isoforms for responsiveness to chemotherapy and survival in ovarian cancer: evidence for a crucial p53-p73 cross-talk in vivo. Clin Cancer Res 11: 8372-8383, 2005

31. Slade N and Moll UM: p53 protocols. Vol 234. Humana Press, Totowa, New Jersey, 2003.

32. Wen WH, Reles A, Runnebaum IB, Sullivan-Halley J, Bernstein L, Jones LA, Felix JC, Kreienberg R, El-Naggar A and Press MF: p53 mutations and expression in ovarian cancers: correlation with overall survival. Int J Gynecol Pathol 18: 29-41, 1999.

33. Alkushi A, Lim P, Coldman A, Huntsman D, Miller D and Gilks CB: Interpretation of p53 immunoreactivity in endometrial carcinoma: establishing a clinically relevant cut-off level. Int J Gynecol Pathol 23: 129-137, 2004.

34. Marchenko ND and Moll UM: Nuclear overexpression of p53 protein does not correlate with gene mutation in primary peritoneal carcinoma. Hum Pathol 28: 1002-1006, 1997.

35. Meinhold-Heerlein I, Ninci E, Ikenberg H, Brandstetter T, Ihling C, Schwenk I, Straub A, Schmitt B, Bettendorf H, Iggo R and Bauknecht T: Evaluation of methods to detect p53 mutations in ovarian cancer. Oncology 60: 176-188, 2001

36. Moll UM, Valea F and Chumas J: Role of p53 alteration in primary peritoneal carcinoma. Int J Gynecol Pathol 16: 156-162, 1997.

37. Wang A and Gehan EA: Gene selection for microarray data analysis using principal component analysis. Stat Med 24: 2069-2087, 2005

38. Lee EH, Chun M, Kang S and Lee HJ: Validation of the functional assessment of cancer therapy-general (FACT-G) scale for measuring the health-related quality of life in Korean women with breast cancer. Jpn J Clin Oncol 34: 393-399, 2004.

39. Frank E, Hall M, Trigg L, Holmes G and Witten IH: Data mining in bioinformatics using Weka. Bioinformatics 20: 2479-2481, 2004

40. Liu JJ, Cutler G, Li W, Pan Z, Peng S, Hoey T, Chen L and Ling XB: Multiclass cancer classification and biomarker discovery using GA-based algorithms. Bioinformatics 21: 2691-2697, 2005

41. Bidart JM, Thuillier F, Augereau C, Chalas J, Daver A, Jacob N, Labrousse $\mathrm{F}$ and Voitot $\mathrm{H}$ : Kinetics of serum tumor marker concentrations and usefulness in clinical monitoring. Clin Chem 45: 1695-1707, 1999.

42. Gadducci A, Cosio S, Fanucchi A, Negri S, Cristofani R and Genazzani AR: The predictive and prognostic value of serum CA 125 half-life during paclitaxel/platinum-based chemotherapy in patients with advanced ovarian carcinoma. Gynecol Oncol 93: 131-136, 2004.

43. Guppy AE and Rustin GJ: CA125 response: can it replace the traditional response criteria in ovarian cancer? Oncologist 7: 437-443, 2002.

44. Irwin MS, Kondo K, Marin MC, Cheng LS, Hahn WC and Kaelin WG Jr: Chemosensitivity linked to p73 function. Cancer Cell 3: 403-410, 2003.

45. Wei JS, Greer BT, Westermann F, Steinberg SM, Son CG, Chen QR, Whiteford CC, Bilke S, Krasnoselsky AL, Cenacchi N, Catchpoole D, Berthold F, Schwab M and Khan J: Prediction of clinical outcome using gene expression profiling and artificial neural networks for patients with neuroblastoma. Cancer Res 64: 6883-6891, 2004.

46. Williams RD, Hing SN, Greer BT, Whiteford CC, Wei JS, Natrajan R, Kelsey A, Rogers S, Campbell C, Pritchard-Jones K and Khan J: Prognostic classification of relapsing favorable histology Wilms tumor using cDNA microarray expression profiling and support vector machines. Genes Chromosomes Cancer 41: 65-79, 2004. 